\title{
Procedure for the transferability of application-specific boundary conditions for the testing of components and products
}

\author{
Lukas Schwan $^{1 *}$, Philipp Hüttich ${ }^{1}$, Marie Wegner ${ }^{1}$, Dieter Krause ${ }^{1}$ \\ ${ }^{1}$ Institute of Product Development and Mechanical Engineering Design (PKT), Hamburg University of Technology \\ (TUHH), Germany \\ * Corresponding author: \\ Lukas Schwan \\ Hamburg University of Technology (TUHH) \\ Institute of Product Development and Mechanical Engineering Design (PKT) \\ Denickestraße 17 \\ D-21073 Hamburg \\ Phone: +4940 / 428782661 \\ Mail: lukas.schwan@tuhh.de
}

\begin{abstract}
Testing plays an important role in the product development process in order to verify the safety and functionality of products and components. Application-specific boundary conditions must be transferred between different model layers and structural complexity levels in order to obtain a close representation of reality. Hereby the view on the overall test system as well as the superordinate problem definition is made difficult for the product developer. In this article a procedure is presented, which builds up on the product component test pyramid. The application of the procedure is shown in the contribution for the virtual testing of sandwich structures, the development of physical models in medical radiotherapy as well as the dynamic testing with combined environmental conditions.
\end{abstract}

\section{Keywords}

Testing Approach, Boundary Conditions, Virtual Testing, Combined Environmental, Radiotherapy Phantom 


\section{Introduction}

In the product development process, testing plays an important role in proving the safety and functionality of products and components. The real system on the targeted complexity level and the loads acting on it are abstracted in tests in the form of a physical test model. Since the costs and effort required to perform such real tests, especially at the product and component level, are very high, virtual test procedures are increasingly used to minimize the test effort. In addition to the model layers mentioned, there are also various levels for categorizing structural complexity. This multitude of different levels, between which boundary conditions must be exchanged during testing, makes it difficult for the product developer to maintain a view of the overall test system as well as the superordinate testing problem. Especially when application-specific boundary conditions, which are not purely mechanical, are transferred, this problem is further increased.

\section{State of the art}

One approach widely used in the aircraft industry to test products and components is the Building Block Approach [1]. In this approach so-called building blocks are defined as structural complexity levels, which are built up from the constituent level in order to reach the product level. This idea of successive, hierarchical model and test design, which builds up on findings from lower structural complexity levels, originates from multiscale analysis, which is widely used in materials science [2]. Breuer [3] uses the complexity levels from the Building Block Approach [1] in his test pyramid and separates them into generic and product-specific complexity levels. Based on the Building Block Approach [1], Krause et al. [4] define a test pyramid with the structural complexity levels of material, structure, sub-product and product level. Figure $1^{1}$ shows the test pyramid according to Heyden et al. $[5,6]$ with the complexity levels of the material, structure and component as well as the product level. They divide the pyramid into a static and a dynamic area, because especially for the transfer of mechanical boundary conditions in lightweight structures it has to be distinguished which type of load is present. Furthermore, they extend the pyramid to include reality as well as physical and virtual test models as model layers. Hartwich et al. [7] also extend the pyramid by the consideration of uncertainties for the designing of lightweight structures.

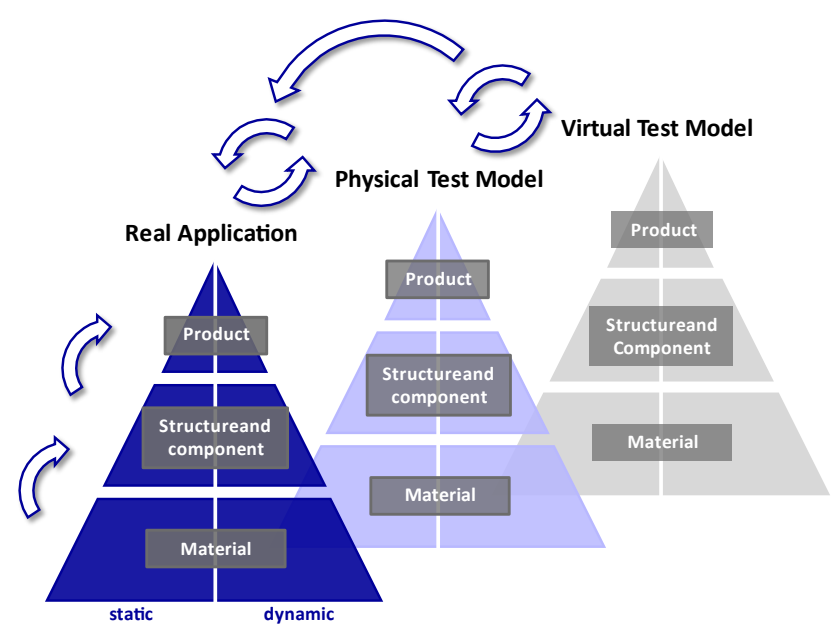

Figure $1^{1}$ : Approach for the transferability of mechanical boundary conditions in lightweight structures according to Heyden et al. [5]

\footnotetext{
1 The original figure is in German and has been translated to English by the authors
} 
The physical test model can, for example, be a test of the real system. To reduce the physical test effort, virtual tests are also used. According to Cox et al. [8], hierarchically structured virtual models validated on real tests are used to describe the behavior of the target system. An approach for virtual testing of sandwich panel joints was developed by Seemann [9,10]. It is a bottom-up approach based on the Building Block Approach [1], where the virtual models are validated on physical tests and synthesized at higher levels of complexity. The combination of physical and virtual models generates synergistic effects which help to gain a better understanding of the system behavior as well as the failure mechanisms. According to Stachowiak [11], both the physical and the virtual models represent an abstraction of the real system according to the abbreviation feature. The purpose of the models (pragmatic feature) is application specific. In order to approximate the behavior of the real system with the help of corresponding test models as realistically as possible, the boundary conditions must be transferred accordingly into the corresponding test level.

\section{Procedure for the transferability of application-specific boundary conditions}

Heyden et al. [5] have shown with their approach how mechanical boundary conditions can be transferred between the different complexity levels and model layers during testing. However, they only refer to lightweight structures, whereas the problems also exist in other application areas in the field of product development and testing. In these it can be necessary to transfer further application-specific, not purely mechanical boundary conditions, such as temperature or humidity boundary conditions. The procedure presented in this paper answers the research question how such application-specific boundary conditions can, in addition to mechanical boundary conditions, also be transferred between the different test levels for the testing of components and products.

The developed procedure is based on the hierarchical product component test pyramid, which is structured according to Heyden et al. [5]. The different model layers are synthesized in a single pyramid in order to visualize the coherence of the model layers more strongly. Also, an explicit division of the pyramid into a static and dynamic part was omitted, since this is only of minor significance in some applications, such as the building of physicals models in medical imaging technology. The structural complexity levels were defined as the material level, the structure and component level and the product level. Since this classification is strongly dependent on the respective field of application as well as the defined system boundaries, an explicit classification of the complexity level cannot be made on a generic level. The defined complexity levels therefore serve as an initial orientation and the exact classification must be made and extended if required by the product developer, taking into account his specific application. For example, a finely granulated subdivision of the structure level into the component, substructure and structure level is conceivable.

Figure 2 illustrates the basic procedure by means of an explanatory example. On the lefthand side, the product component test pyramid is first shown in its 3D initial form with the defined structural complexity levels and the top view of the pyramid is shown on the right. The test task in the example is to build a virtual test model on component level. For this purpose, the structure and component levels could first be divided into the corresponding levels. In order to remain with the basic representation of the test pyramid this is renounced in the explanation example. Afterwards the product developer arranges itself with its task in the product component test pyramid, which is realized by the flag symbol in the top view of the pyramid. The application-specific boundary conditions are then transferred from the corresponding complexity levels and model layers. In the selected example, these are mechanical boundary conditions from a physical test model on product-level (1) and temperature boundary conditions from the real system (2). While the numbers are only used as a reference for the test levels involved, the colors of the arrows indicate which type of boundary condition is transferred. The types of the occurring boundary condition, which are transferred, can be 
adapted to the respective application. The direction of transfer of the boundary condition has to be selected in the direction of the target level, whereby the transferability should always be given in both directions for taking into account iteration processes for example. To represent cross-sectional tasks between different model layers, in which synergetic effects are accessible, it is possible to use the intersection line of two model layers. Corresponding examples for this use case are given in chapter 4.

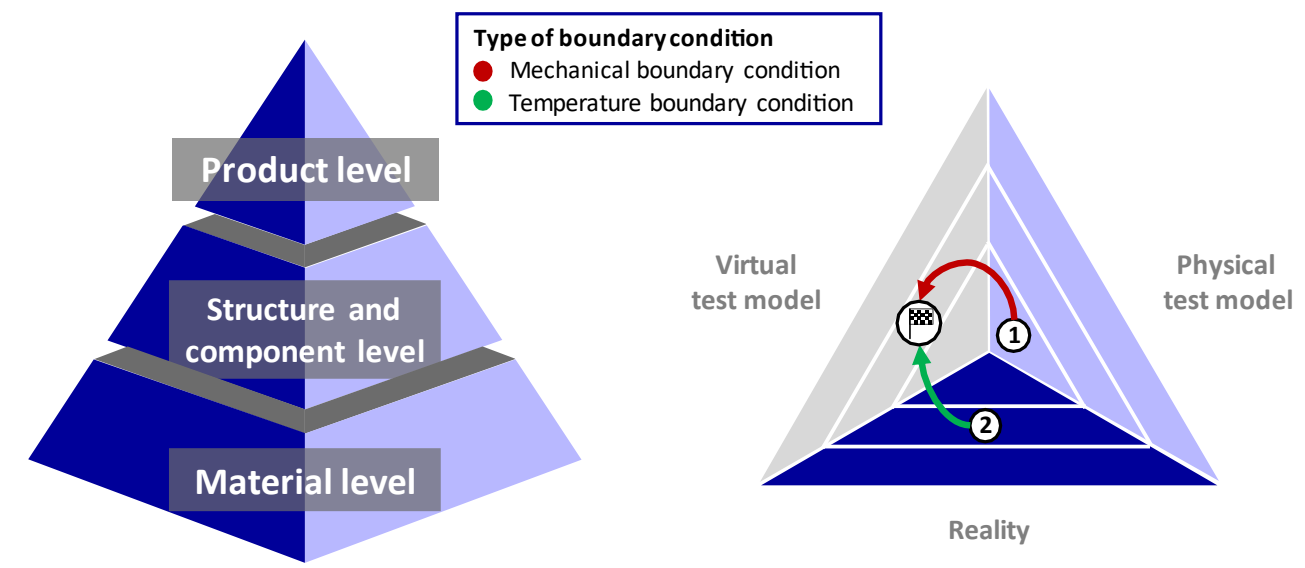

Figure 2: Procedure for the transferability of application-specific boundary conditions based on the product component test pyramid

\section{Application examples of the procedure}

In various application areas in the field of product development, there are challenges and various hurdles in the transfer of application-specific boundary conditions. This chapter describes challenges in the application areas of virtual testing of sandwich structures, dynamic testing with combined environmental conditions and physical modeling in medical imaging technology and afterwards shows how the presented procedure can be applied in these areas.

\subsection{Virtual testing of sandwich structures on substructure level}

In the following paragraph, the product component test pyramid is applied for virtual testing of sandwich structures on substructure level. Based on this application example, it is shown that the presented procedure is also suitable for challenges in the area of transferability of purely mechanical boundary conditions.

\subsubsection{Initial situation}

Due to their very good weight-specific material properties, sandwich structures are often used in aircraft design [12,13]. The approval is carried out according to the CS-25 guideline [14], whereby a full-size test must always be carried out at the product level. In order to minimize the real test effort, especially at lower structural complexity levels, virtual test procedures based on the finite element method (FEM) are used [15-17]. The critical areas in which local failure mostly occurs in the sandwich structures are the connecting elements, which can be ranked at component level in the product component test pyramid $[9,10]$. For this reason, the Insert Design Handbook [18] recommends various tests, such as the pull-out test for testing inserts perpendicular to the face sheet. Using the virtual testing approach for sandwich panel joints developed by Seemann $[9,10]$, it is possible to create virtual models of these component tests to predict the strengths and progressive failure mechanisms of the sandwich structures. The described tests are performed under quasi-static load and the 
application-specific boundary conditions in this area are purely mechanical. In the following, it will be shown that the presented procedure can also be applied to such problems.

\subsubsection{Problem and objectives}

An existing problem of tests on component level is, that the boundary conditions in these tests are only represented in an idealized way. For example, in the pull-out test mentioned above, a round fixture with a diameter of $70 \mathrm{~mm}$ is suggested in the Insert Design Handbook [18]. While such tests are suitable for determining strengths and local failure mechanisms at the inserts, they reach their limits especially when representing the partially global system and stiffness behavior. According to the Building Block Approach [1], after considering the component level, it is suggested to shift directly to the product level. However, this structural complexity level shift is huge and unsuitable for building up virtual models on product level. In this case there is a huge number of influencing parameters, which have to be taken into account and investigated on intermediate structural complexity levels as well as validated in corresponding models first. In particular, there is a lack of appropriate test setups for building up models on these intermediate structural levels, which take into account the partially global stiffness and system behavior of sandwich structures in addition to local failure mechanism. The problem arises, how the application-specific mechanical boundary conditions in such virtual tests, consisting of a virtual test model and a physical test model for validation purposes have to be transferred.

\subsubsection{Application of the procedure}

The application of the presented procedure to the described problem of virtual testing of sandwich structures is shown in Figure 3. In order to obtain an intermediate level between product and component level, for the specific use case the structure and component level of the generic product component test pyramid can be divided into the component level and the substructure level. The aim is to create a virtual test at substructure level, which also takes into account the partially global system behavior of the sandwich structure. Since a virtual test (1) is the synergetic combination of a real test and its virtual representation, the boundary line (black) of the pyramid between the virtual and the physical test model can be selected.

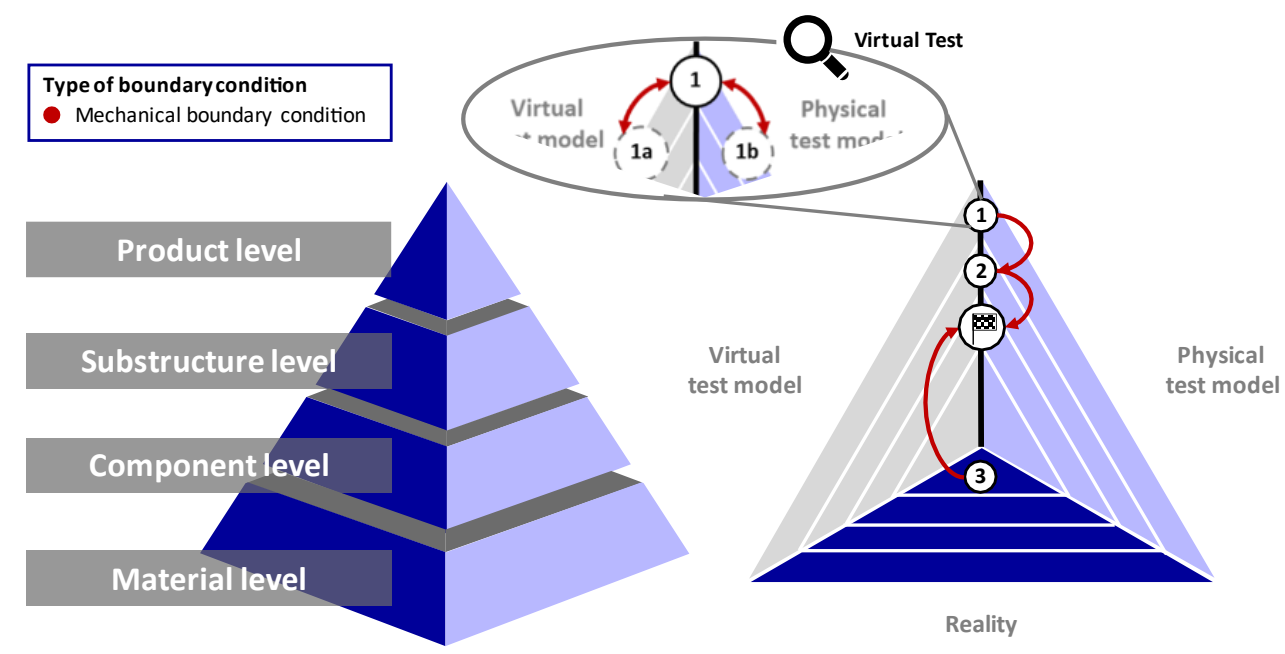

Figure 3: Application of the procedure for the formulating of a virtual test for sandwich structures on substructure level

The exchange of boundary conditions in the case of a virtual test occurs in both directions of virtual (1)a and physical test model (1) $b$ at the same structural complexity level, as shown 
in the enlarged section of the figure. The findings from lower levels, such as material parameters or information regarding the level of detail, are then transferred to the next higher structural complexity level (2) up to the target level using a bottom-up approach. To obtain a more realistic representation of the boundary conditions at the system boundaries in the substructure test, the mechanical boundary conditions from the real system at product level (3) are transferred to substructure level using a top-down approach. This approach is based on the Wishbone approach according to Ostergaard [15], in which a bottom-up and a top-down approach are synthesized to obtain a corresponding complexity level from two directions in order to take into account local and partially global behavior.

\subsection{An approach for testing under combined environmental conditions}

In this chapter, an overview of testing with combined environmental conditions is given and the challenges for developing a corresponding physical test model are presented. With the help of the product component test pyramid, the interrelationships of the individual environmental conditions and the transfer between the individual layers and complexity levels are explained.

\subsubsection{Initial situation}

In aviation-specific product development, the function of a product is secured by the certification process, for which realistic tests are necessary. Apart from HALT (highly accelerated lifetime) tests with less realistic conditions for rapid damage [19], current test procedures in aviation are designed to test environmental conditions predominantly only individually. The best known of these influences are vibration, pressure, temperature and humidity. Common tests, especially in the automotive industry according to OEM, only combine temperature changes with different vibration curves [20]. In aviation, it is permissible to use alternative procedures as a combination of the individual certifications, provided that it can be demonstrated, that all applied environmental conditions specified in the original procedures are replicated or exceeded in the combined procedure [21].

Combined or superimposed environmental testing is the simultaneous impact of multiple stress factors on a test object [22]. The combinations of environmental conditions should be chosen to ensure the electrical and mechanical functions of the test specimen during storage, transport and operation with respect to safety, integrity and performance [23]. Although it is impossible to replicate the complex mix of all environments at the same time, the intent is to apply representative combinations of stresses to the material to determine performance and capabilities [24]. In principle, separating the environmental influences into different individual tests is permissible [21] and reasonable in order to be able to clearly assign the failure to the respective stress factors without having to make concessions in the test result. In the case of environmental conditions with strong interaction, an exact statement is only possible to a limited extent, such as in the case of mechanical-thermal or climatic-thermal stresses [22]. The synergetic effects caused by the combination can lead to failures that would not occur in individual environmental tests [22,24]. Accordingly, an increase in reliability as well as holistically more realistic loads can be achieved.

\subsubsection{Problem and objectives}

The difficulty with investigations on test rigs is the realistic modelling of the interactions between the test specimen and the surrounding systems. This applies to the mechanical boundary conditions such as vibrations or pressure, as well as other boundary conditions like temperature and humidity. In the development process, the boundary conditions and limits of the test environment can be estimated with the help of virtual FEM models and weaknesses 
of the product can be predicted. For a systematic approach, a hierarchy of test levels according to the product component test pyramid is useful in order to keep the testing effort and associated costs low. Tests at the material level require less effort and therefore allow a higher number of tests. Subsequently, tests are to be carried out at the structure level and for the entire product, for which increasing effort and therefore a reduced number of tests are to be expected.

\subsubsection{Application of the procedure}

The application of the product component test pyramid to the problem of testing with combined environmental conditions is described in Figure 4. The mechanical, thermal and hydrological boundary conditions are specified by the certification standard, which corresponds to the boundary conditions of the real system at product level (1). For the step-by-step construction of a physical and virtual model, it is necessary first to transfer the boundary conditions to the material level (2), whereby this is only done for temperature and humidity using a top-down approach. The mechanical conditions cannot be applied the material level in the same way as at the product level. Similar to the procedure described in chapter 4.1.3, an exchange of boundary conditions between the physical and virtual model on material level takes place first. In this way, a validated simulation model can be set up while the function of the physical test model can be tested and ensured due to limited complexity. With the knowledge gained at material level, mechanical boundary conditions can be applied with the bottom-up approach to the next higher structure and component level (3), whereby the boundary conditions for temperature and humidity are again specified by the real system at product level. After tests with the structure and component parts have been successfully carried out at this level and a validated simulation model has been set up, it is possible to transfer the mechanical boundary conditions to the product level (4) of the virtual test model. It should be noted, that in addition to temperature and humidity, mechanical boundary conditions of the real system must also be taken into account in this case. As described in chapter 4.1, however, the high increase of complexity from the structure to the product level poses a major challenge. From this model, information can then be drawn, particularly on the mechanical boundary conditions, which can be incorporated into the development of a physical test model at product level.

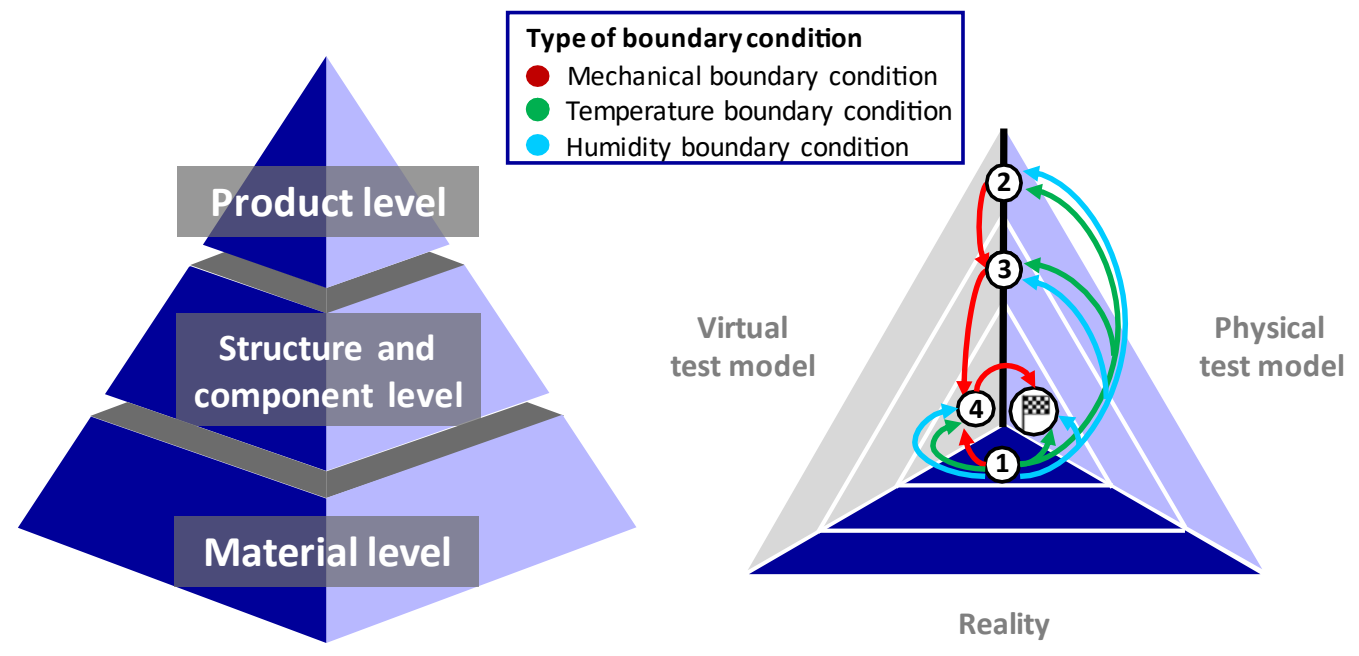

Figure 4: Application of the procedure for testing with environmental boundary conditions 


\subsection{Testing in radiotherapy using phantoms with integrated biological samples}

In the following chapter the product component test pyramid is applied on physical testing of imaging models used in the preclinical medical radiotherapy. Throughout this it is shown that the presented procedure is also suitable in different application fields and conditions.

\subsubsection{Initial situation}

Using radiotherapy, tumor cells are killed with the help of a high dose of radiation, while at the same time the surrounding normal tissue must be protected as best as possible. Research into novel treatment approaches in radiotherapy can be carried out in preclinical studies with sophisticated small animal radiation platforms. Preclinical studies have a central role towards improving patient outcomes and the ability to accurately model biological and physical aspects, which in radiation oncology is critical to achieve [25]. Using models, the response of a therapy or probabilities of side effects can be better predicted and thus in the long term an individualization of the treatment concept can be made possible. In order to ensure optimal use and correct mimicking of clinical radiotherapy treatments, adequate quality assurance methods should be established for the preclinical irradiation platforms. This concerns on the one hand physical radiotherapy models, so called phantoms, which have the same physical properties in irradiation and imaging of small animals and are therefore suitable for use in dosimetry $[26,27]$. Secondly, virtual models, using e.g., Monte Carlo simulations, are needed to calculate irradiation plans and the expected dose [26].

\subsubsection{Problem and objectives}

To keep the number of small animal experiments for preclinical radiation to a minimum, while maintaining the realistic properties of small animals, accurate phantoms need to be constructed. But physical phantoms still can't represent the biological responses of irradiated tissue. Thus, a new approach is to integrate real-life substructure, such as 3D cell matrices or tissue samples, into a physical small animal phantom. This expands the ability to accurately model biological as well as physical aspects in advanced preclinical radiotherapy research. By using biological tissue inside a phantom, it is possible to study the entire therapy process on a physical model. This can help reducing the usage of test animals while gaining knowledge regarding the diagnostics and the course of therapy of tumor diseases.

A step-by-step testing approach to generate a phantom is needed to bridge the different test models and levels as well as their boundary conditions together. Virtual simulation of the radiation is needed to characterize the physical model for the radiotherapy. For the phantom development it is important to start at material level and then implement results into the next complexity level, with the final goal being a phantom with integrated real tissue samples.

\subsubsection{Application of the procedure}

Figure 5 shows the approach applied in the product component test pyramid for the testing of new radiotherapy approaches on small animal phantoms with the integration of biological samples. The development consists of two main steps. First, the material level is investigated (1). From the real plane of small animal tissue materials, the radiotherapy properties, like tissue equivalent mass density and effective atomic number, for the physical phantom are determined (1)a. The possible phantom materials are tested and adapted together with a virtual tissue model (1) b, resulting in a dependency of the virtual model and the phantom.

After the lower material level is determined, the information from this level is applied using the bottom-up approach to the higher complexity levels (2). This is being done for the physical 
phantom. Like in the lower-level information for the real structure is transferred into the phantom (2)a. Furthermore, the simulation of the structure is again also performed and adapted (2)b. This results in (2), which has a small animal phantom as output. For the final goal on component level tumor samples or cell samples (2)a, that represent the real biological aspects, are taken and integrated in the physical small animal phantom. Resulting in the final target level linking the real and physical model on the highest level.

In this approach all layers of the three-dimensional test pyramid are used. Real properties are firstly transferred into the physical phantom and from this plane applied and adapted together with the virtual model. Finally, special care was applied to conducting experiments that bridge real structures, such as cell or tissue samples, with physical phantoms, representing anatomical properties. Thus combining in vivo material with in vitro stetting, while observing radiation properties through dose simulation.

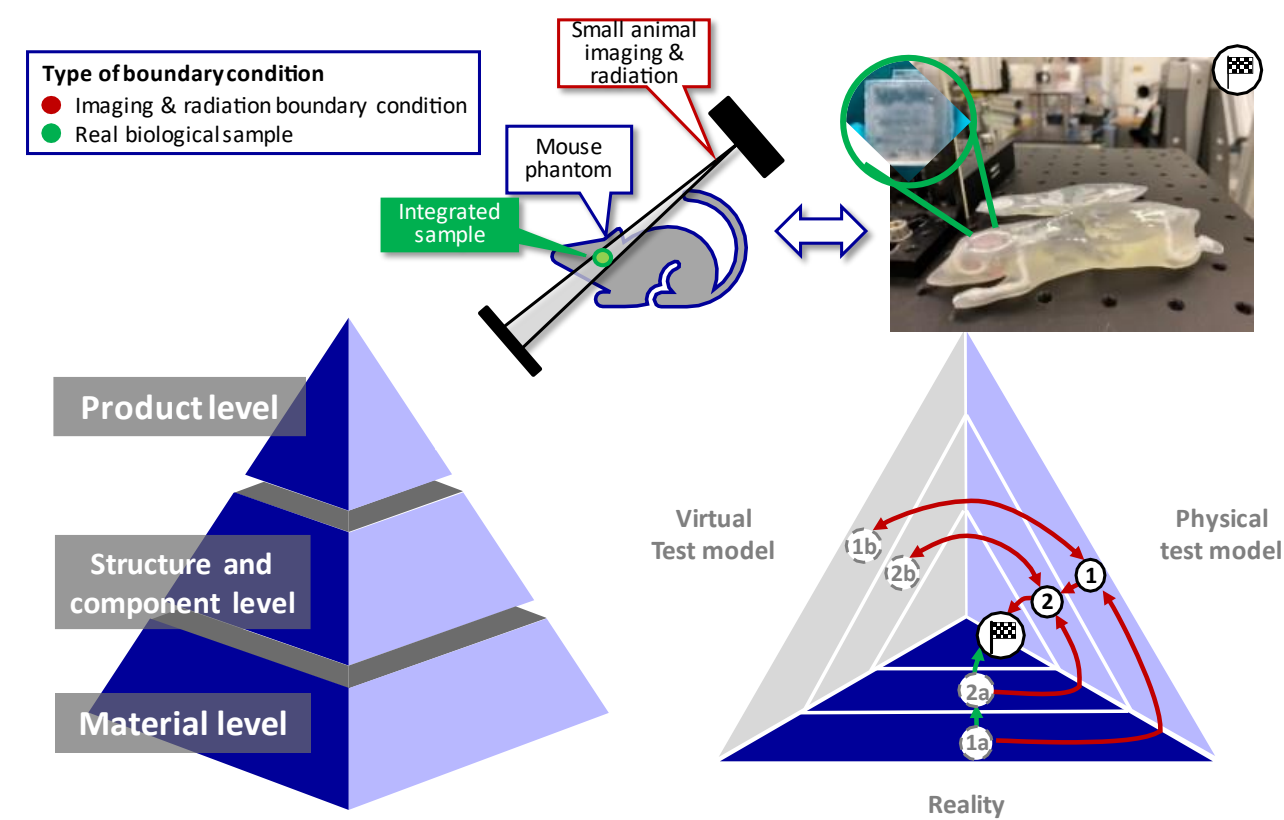

Figure 5: Application of the procedure for the integration of biological samples into a physical small animal phantom for preclinical radiotherapy

\section{Summary and Outlook}

The transferability of application-specific boundary conditions between different structural complexity levels and model layers presents product developers with the challenge of maintaining a view of the overall test system and the superordinate problem definition when testing products and components. This paper presented a procedure based on the product component test pyramid, that enables the transferability of application-specific boundary conditions. The product component test pyramid consists of the model layers reality, physical and virtual test model as well as the complexity levels of the material, component and product level. The different levels are synthesized into a single pyramid. The transferability of the boundary conditions in the pyramid is realized via colored arrows. The application of the procedure has been demonstrated for the virtual testing of sandwich structures, physical models in medical imaging technology as well as the combined environmental testing.

\section{Acknowledgements}

Parts of this contribution were supported by the LuFo VI-1 projects "Effiziente Kabine durch digitale Vernetzung von Technologien und Systemen (EFFEKT)" and "New Cost-Effektive \& Reliable Test Environments (Certev)" funded by the Federal Ministry for Economic Affairs and 
Energy (BMWi). Further parts of this contribution are based on a project funded by the Behörde für Wissenschaft, Forschung und Gleichstellung (BWFG) as subproject within the framework of the Graduate School "Innovative Technologies in Cancer Diagnostics and Therapy".

\section{References}

[1] Department of Defense: Composite Materials Handbook. Volume 3. Polymer Matrix Composites Materials Usage, Design, and Analysis. Washington: Department of Defense, 2002.

[2] Okereke, Michael I.; Akpoyomare, Ambrose; Bingley, Mark: Virtual testing of advanced composites, cellular materials and biomaterials: A review. In: Composites Part B: Engineering (2014), No. 60, pp. 637-662.

[3] Breuer, Ulf P.: Commercial Aircraft Composite Technology. Basel: Springer International Publishing, 2016.

[4] Krause, Dieter et al.: Produktvalidierung von Leichtbaustrukturen für die Flugzeugkabine. In: 65 . Deutscher Luft- und Raumfahrtkongress. Braunschweig, 2016.

[5] Heyden, Emil et al.: Aktuelle Ansätze in der Entwicklung und Auslegung von Leichtbaustrukturen. In: Krause, Dieter; Hartwich, Tobias S.; Rennpferdt, Christoph (Eds.): Produktentwicklung und Konstruktionstechnik Forschungsergebnisse und -projekte der Jahre 2016 bis 2020, Berlin: Springer, 2020.

[6] Heyden, Emil et al.: Transferability of Boundary Conditions in Testing and Validation of Lightweight Structures. In: Proceedings of the 30th Symposium Design for X (DfX 2019), Jesteburg, 2019, pp. 85-96.

[7] Hartwich, Tobias S. et al.: Designing Lightweight Structures under Consideration of Material and Structure Uncertainties on Different Levels of the Building Block Approach. In: Proceedings of the 31th Symposium Design for X (DfX 2020), Erlangen, 2020, pp. 121-130.

[8] Cox, Brian N.; Spearing, S. Mark; Mumm, Daniel R.: Practical Challenges in Formulating Virtual Tests for Structural Composites. In: Camanho, Pedro P. et al. (Eds.): Mechanical Response of Composites. Dordrecht, Springer Netherlands, 2008, pp. 57-75.

[9] Seeman, Ralf: A Virtual Testing Approach for Honeycomb Sandwich Panel Joints in Aircraft Interior. Technische Universität Hamburg, Hamburg, 2019.

[10] Seeman, Ralf; Krause, Dieter: Virtual Testing of Nomex Honeycomb Sandwich Panel Inserts. In: Proceedings of the 20th International Conference on Composite Materials. Kopenhagen, 2015.

[11] Stachowiak, Herbert: Allgemeine Modelltheorie. Wien: Springer, 1973.

[12] Zenkert, Dan: The handbook of sandwich construction. Cradley Heath, West Midlands: Engineering Materials Advisory Services Ltd. (EMAS), 1997.

[13] Bitzer, Tom: Honeycomb technology - Materials, design, manufacturing, applications and testing. London, New York: Chapman \& Hall, 1997.

[14] European Aviation Safety Agency, 2007. Certification Specifications for Large Aeroplanes CS-25.

[15] Ostergaard, Morten G. et al.: Virtual testing of aircraft structures. In: CEAS Aeronautical Journal (2011), No. 1, pp. 83-103.

[16] Seemann, Ralf; Krause, Dieter: Numerical modelling of partially potted inserts in honeycomb sandwich panels under pull-out loading. In: Composite Structures (2018), No. 203, pp. 101-109.

[17] Heimbs, Sebastian; Pein, Marc: Failure behaviour of honeycomb sandwich corner joints and inserts. In: Composite Structures (2009), No. 89; pp. 575-588.

[18] ESA Requirements and Standards Division: Space Engineering: Insert design handbook. ECSS Secretariat, Netherlands, 2011.

[19] Raheja, Dev; Guello, Louis J.: Design for Reliability. New Jersey: John Wiley \& Sons, Inc., 2012

[20] Norm VW 80000, Elektrische und elektronische Komponenten in Kraftfahrzeugen bis 3,5t - Allgemeine Anforderungen, Prüfbedingungen und Prüfungen, 2013.

[21] RTCA, Inc.: RTCA/DO160G - Environmental Conditions and Test Procedures for Airborne Equipment. Washington, 2010.

[22] Vogl, Günter: Umweltsimulation für Produkte. Würzburg: Vogel Verlag und Druck GmbH \& Co. KG,1999

[23] Norm DIN EN 60068-2-53, Umgebungseinflüsse - Teil 2-53: Prüfverfahren - Prüfungen und Leitfaden Kombinierte klimatische (Temperatur/Luftfeuchte) und dynamischer (Schwingung/Schock) Prüfungen,2011.

[24] Thompson, Ken et al.: MIL-STD-810H - Department of Defense Test Method Standard - Environmental Engineering Considerations and Laboratory Tests. Washington, 2019.

[25] Ghita, Mihaela et al.: Integrating Small Animal Irradiators withFunctional Imaging for Advanced Preclinical Radiotherapy Research. In: Cancers (Basel), No. 11, 170, 2019.

[26] Biglin, Emma R., et al.: Preclinical dosimetry: exploring the use of small animal phantoms, In: Radiat Oncology, No. 14, 134, 2019.

[27] Spallek, Johanna et al.: Entwicklung und Anwendung medizinischer Simulationsmodelle. In: Krause, Dieter et al. (Ed.): Produktentwicklung und Konstruktionstechnik, Forschungsergebnisse und -projekte der Jahre 2016 bis 2020, Springer Verlag, Berlin, Heidelberg, Germany, pp. 255-272, 2020. 